\title{
Some relations concerning triangles and bicentric quadri- laterals in connection with Poncelet's closure theorem when conics are circles not one inside of the other
}

\author{
Mirko Radić
}

M. Radić studied mathematics at the University of Zagreb, where he was primarily trained as an algebraist. Presently, he is professor emeritus at the University of Rijeka, Croatia. There he was lecturing for more than fourty years. He is still active and working on problems concerning polygons.

\section{Introduction}

A polygon which is both chordal and tangential will be called a bicentric polygon. The first who was concerned with bicentric polygons was the German mathematician Nicolaus Fuss (1755-1826), a friend of Leonhard Euler (see [5]). He posed the following problem (known as Fuss' problem of the bicentric quadrilateral):

Find the relation between the radii and the line segment joining the centres of the circles of circumscription and inscription of a bicentric quadrilateral.

He found that

$$
2 \rho^{2}\left(r^{2}+z^{2}\right)=\left(r^{2}-z^{2}\right)^{2},
$$

where $r$ and $\rho$ are radii and $z$ is the distance between the centers of the circles of circumscription and inscription.

Die allgemeine Fassung des Schliessungssatzes von Poncelet besagt folgendes: Formen $C, C_{1}, \ldots, C_{n}$ ein Kegelschnittbüschel, ist $P \in C$ ein Punkt, konstruiert man $P_{1}, \ldots, P_{n} \in C$ derart, dass die Gerade durch $P P_{1}$ die Kurve $C_{1}$, die Gerade durch $P_{1} P_{2}$ die Kurve $C_{2}, \ldots$, die Gerade durch $P_{n-1} P_{n}$ die Kurve $C_{n}$ berührt und entsteht bei dieser Konstruktion die Gleichheit $P=P_{n}$, so gilt diese Koinzidenz unabhängig von der Wahl von $P$. In der vorliegenden Arbeit werden die Spezialfälle $n=3$ und $n=4$ betrachtet, wobei zusätzlich vorausgesetzt wird, dass die Kegelschnitte (nicht notwendigerweise verschiedene) Kreise sind. In den genannten Spezialfällen, in denen zudem die Kreise nicht ineinander enthalten sind, wird ein elementarer Beweis des Satzes von Poncelet gegeben. 
This problem is listed and considered in [4, p. 188] as one of the 100 great problems of elementary mathematics.

Fuss also found corresponding formulas for bicentric pentagons, hexagons, heptagons and octagons (Nova Acta Petropol., XII, 1798).

The corresponding formula for triangles is

$$
r^{2}-z^{2}=2 r \rho
$$

and had already been given by Euler.

The very remarkable theorem concerning bicentric polygons is given by the French mathematician Poncelet (1788-1867). In the formulation of this theorem the so-called Poncelet traverse will be used. This in short is:

Let $C_{1}$ and $C_{2}$ be two circles in a plane. If from any point on $C_{2}$ we draw a tangent to $C_{1}$, extend the tangent line so that it intersects $C_{2}$, and draw from the point of intersection a new tangent to $C_{1}$, extend this tangent similarly to intersect $C_{2}$, and continue in this way, we obtain the so-called Poncelet traverse which, when it consists of $n$ chords of the circle $C_{2}$ (circle of circumscription), is called $n$-sided.

The Poncelet theorem for circles can be expressed as follows:

If on the circle of circumscription there is one point of origin for which the nsided Poncelet traverse is closed, then the n-sided traverse will also be closed for any other point of origin on the circle.

Poncelet proved that the analogue holds for conic sections so that the general theorem reads:

Poncelet's closure theorem. If an n-sided Poncelet traverse constructed for two given conic sections is closed for one position of the point of origin, it is closed for any position of the point of origin.

Although this problem dates back to the nineteenth century, many mathematicians have been working on a number of problems in connection with it. Many contributions have been made. Very interesting and useful information about this we found in the references concerning Poncelet's closure theorem, particulary in [2], [6] and [8].

In this article we shall restrict ourselves to triangles and bicentric quadrilaterals when the conics are circles not one inside of the other and where instead of incircles there are excircles under consideration. In this case for triangles instead of relation (1.2) Euler's relation holds:

$$
z^{2}-r^{2}=2 r \rho
$$

But Fuss' relation (1.1) holds in both of these cases. (More about this will be given in Section 3.) 


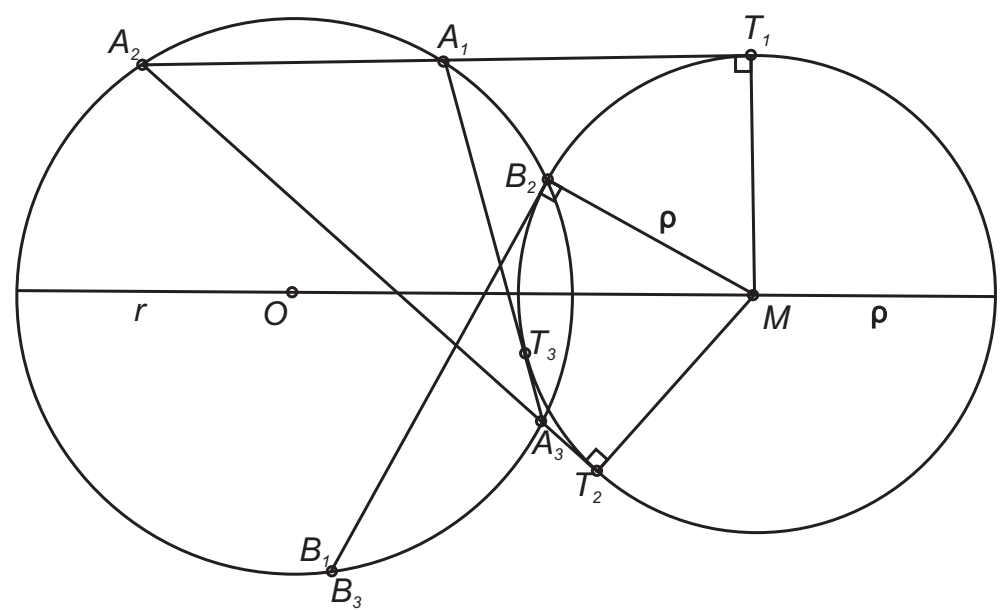

Fig. 1

\section{Some relations concerning triangles which have the same excircle and same circumcircle}

Notation used in this section:

Let $r, z$ and $\rho$ be any given lengths (positive numbers) such that Euler's relation (1.3) holds, and let $M$ and $O$ be points and $C_{1}$ and $C_{2}$ be circles such that

$$
|M O|=z, \quad C_{1}=M(\rho), \quad C_{2}=O(r) .
$$

Then, by Poncelet's closure theorem, for every point $A_{1}$ on $C_{2}$ there is a triangle $A_{1} A_{2} A_{3}$ whose excircle is $C_{1}$ and circumcircle $C_{2}$. (See Fig. 1 , where $r=3, z=5, \rho=\frac{8}{3}$.)

A triangle will be degenerate if one of its vertices belongs to the set $\left\{P_{1}, P_{2}, Q_{1}, Q_{2}\right\}$, where the points $P_{1}, P_{2}, Q_{1}, Q_{2}$ are shown in Fig. 2. So, for example, triangle $B_{1} B_{2} B_{3}$ shown in Fig. 1 is a degenerate one.

Now, let us consider Fig. 3. It is easy to see that

$$
\left(t_{1}-t_{2}-t_{3}\right) \rho=\text { area of triangle } A_{1} A_{2} A_{3},
$$

where $t_{i}=\left|A_{i} T_{i}\right|, i=1,2,3$. Thus, in this case, instead of $t_{2}$ and $t_{3}$ we must take $-t_{2}$ and $-t_{3}$. It is because in this case we must use oriented angles. Namely, if the angle $M A_{i} T_{i}$ is negatively oriented, then instead of $t_{i}$ we must take $-t_{i}$.

It can be easily seen that for every triangle $A_{1} A_{2} A_{3}$ whose excircle is $C_{1}$, one of the angles $M A_{i} T_{i}, i=1,2,3$, is negatively oriented and the other two positively, or conversely, one is negatively oriented and the other two positively.

Also, it is easy to see that

$$
\left|\underline{t}_{i}+\underline{t}_{i+1}\right|=\left|A_{i} A_{i+1}\right|, \quad i=1,2,3,
$$




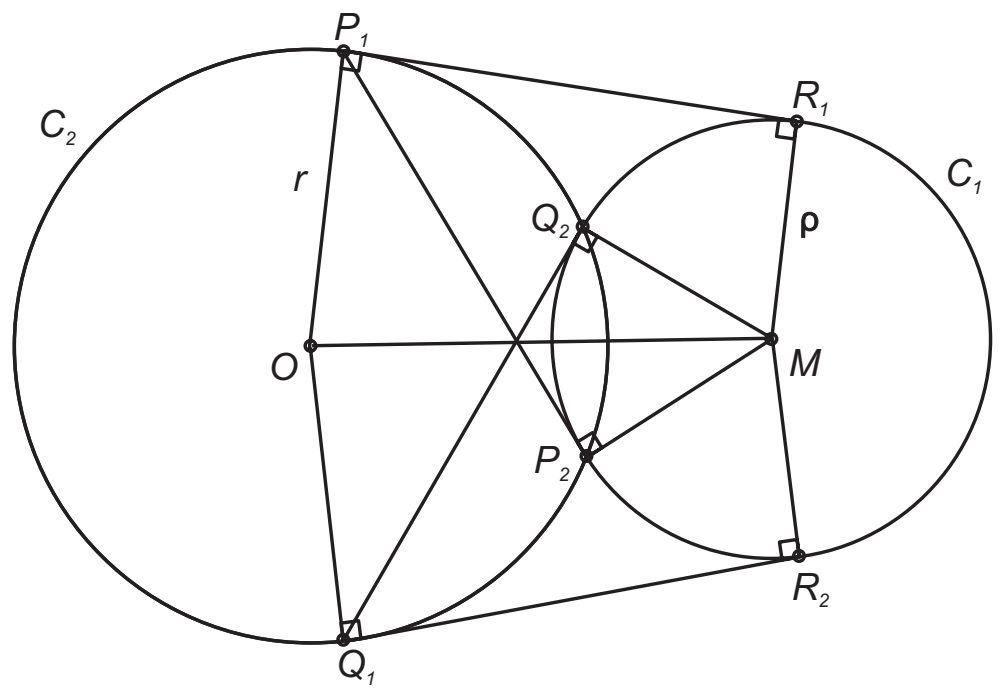

Fig. 2

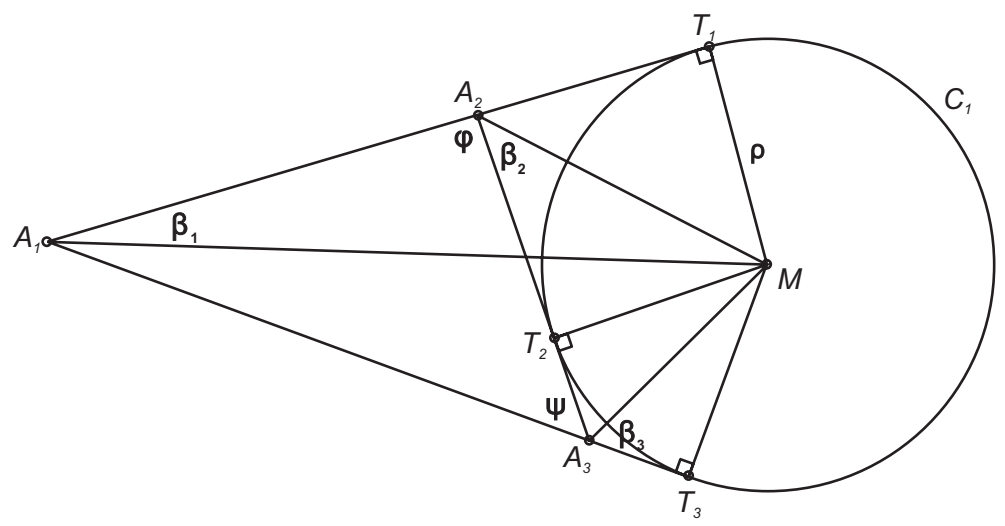

Fig. 3

where

$$
\begin{aligned}
& \underline{t}_{i}=t_{i} \text { if } \angle M A_{i} T_{i} \text { is positively oriented, } \\
& \underline{t}_{i}=-t_{i} \text { if } \angle M A_{i} T_{i} \text { is negatively oriented. }
\end{aligned}
$$

Using vertices $A_{1}, A_{2}, A_{3}$ instead of $T_{1}, T_{2}, T_{3}$ this can be expressed as follows:

$$
\begin{aligned}
& \underline{t}_{i}=t_{i} \text { if } \angle M A_{i} A_{i+1} \text { is positively oriented, } \\
& \underline{t}_{i}=-t_{i} \text { if } \angle M A_{i} A_{i+1} \text { is negatively oriented. }
\end{aligned}
$$

Of course, if $\angle M A_{i} A_{i+1}$ is "obtuse" then its supplement is taken.

Remark 1 For simplicity in some of the formulations in this section we shall assume that the vertices of every triangle $A_{1} A_{2} A_{3}$ whose excircle is $C_{1}$ and circumcircle $C_{2}$ are 
denoted such that

$$
\left|A_{1} M\right|=\max \left\{\left|A_{1} M\right|,\left|A_{2} M\right|,\left|A_{3} M\right|\right\} .
$$

So, for example, triangle $A_{1} A_{2} A_{3}$ in Fig. 3 is such. Triangle $A_{1} A_{2} A_{3}$ in Fig. 1 becomes such if $A_{1}$ and $A_{2}$ are mutually interchanged.

Using Fig. 2 it can be said that $A_{1} \in \widehat{P}_{1} \widehat{Q}_{1}$, where $\overparen{P}_{1} \widehat{Q}_{1} \cap \overline{O M}=\emptyset$. As will be seen, doing so, nothing essentially will be changed. First, it can be easily proved that

$$
\left(t_{1}-t_{2}-t_{3}\right) \rho^{2}=t_{1} t_{2} t_{3} .
$$

Namely, from Fig. 3 we see that

$$
2 \beta_{2}=2 \beta_{1}+\psi, \quad 2 \beta_{3}=2 \beta_{1}+\varphi,
$$

from which we get

$$
-\beta_{1}+\beta_{2}+\beta_{3}=90^{\circ}
$$

Thus, we can write

$$
\begin{aligned}
\cot \left(\beta_{2}+\beta_{3}\right) & =-\tan \beta_{1}, \\
\cot \beta_{1}-\cot \beta_{2}-\cot \beta_{3} & =\cot \beta_{1} \cot \beta_{2} \cot \beta_{3}, \\
\frac{t_{1}}{\rho}-\frac{t_{2}}{\rho}-\frac{t_{3}}{\rho} & =\frac{t_{1} t_{2} t_{3}}{\rho^{3}},
\end{aligned}
$$

which can be written as (2.3). Now, we can prove the following theorem.

Theorem 2.1 For every triangle $A_{1} A_{2} A_{3}$ which is such as described in Remark 1, the following holds:

$$
\left|-t_{1} t_{2}+t_{2} t_{3}-t_{3} t_{1}\right|=4 r \rho-\rho^{2}
$$

Proof. From (2.3) we have

$$
t_{3}=\frac{\rho^{2}\left(t_{1}-t_{2}\right)}{t_{1} t_{2}+\rho^{2}} .
$$

Using the above expression for $t_{3}$ we get

$$
\left|-t_{1} t_{2}+t_{2} t_{3}-t_{3} t_{1}\right|=\frac{\rho^{2}\left(t_{1}^{2}+t_{2}^{2}\right)+t_{1}^{2} t_{2}^{2}-\rho^{2} t_{1} t_{2}}{t_{1} t_{2}+\rho^{2}} .
$$

Now, we can use the relations

$$
J=\left(t_{1}-t_{2}-t_{3}\right) \rho, \quad J=\frac{a b c}{4 r},
$$

where

$$
J=\text { area of } A B C, \quad a=t_{1}-t_{2}, \quad b=t_{2}+t_{3}, \quad c=t_{1}-t_{3} .
$$


From

$$
\left(t_{1}-t_{2}-t_{3}\right) \rho=\frac{\left(t_{1}-t_{2}\right)\left(t_{2}+t_{3}\right)\left(t_{1}-t_{3}\right)}{4 r}
$$

and from (2.6) we get

$$
4 r \rho=\frac{\left(\rho^{2}+t_{1}^{2}\right)\left(\rho^{2}+t_{2}^{2}\right)}{t_{1} t_{2}+\rho^{2}}
$$

or, subtracting $\rho^{2}$ from both sides,

$$
4 r \rho-\rho^{2}=\frac{\rho^{2}\left(t_{1}^{2}+t_{2}^{2}\right)+t_{1}^{2} t_{2}^{2}-\rho^{2} t_{1} t_{2}}{t_{1} t_{2}+\rho^{2}} .
$$

So, equation (2.7) can be written as (2.5). Theorem 2.1 is proved.

Corollary 2.1.1 For every triangle $A_{1} A_{2} A_{3}$ whose excircle is $C_{1}$ and circumcircle is $\mathrm{C}_{2}$

$$
\left|\underline{t}_{1} \underline{t}_{2}+\underline{t}_{2} \underline{t}_{3}+\underline{t}_{3} \underline{t}_{1}\right|=4 r \rho-\rho^{2}
$$

holds, where

$$
\begin{aligned}
& \underline{t}_{i}=t_{i} \text { if } \angle M A_{i} T_{i} \text { is positively oriented, } \\
& \underline{t}_{i}=-t_{i} \text { if } \angle M A_{i} T_{i} \text { is negatively oriented. }
\end{aligned}
$$

Proof. The value $\left|\underline{t}_{1} \underline{t}_{2}+\underline{t}_{2} \underline{t}_{3}+\underline{t}_{3} \underline{t}_{1}\right|$ does not depend upon numeration of vertices of a triangle whose excircle is $C_{1}$ and circumcircle is $C_{2}$.

Corollary 2.1.2 Let $A_{1} A_{2} A_{3}$ and $B_{1} B_{2} B_{3}$ be any two triangles whose excircles have equal radii. Then the circumcircles of these triangles have also equal radii iff

$$
\left|\underline{t}_{1} \underline{t}_{2}+\underline{t}_{2} \underline{t}_{3}+\underline{t}_{3} \underline{t}_{1}\right|=\left|\underline{u}_{1} \underline{u}_{2}+\underline{u}_{2} \underline{u}_{3}+\underline{u}_{3} \underline{u}_{1}\right|
$$

where

$$
\begin{aligned}
\left|\underline{t}_{i}+\underline{t}_{i+1}\right| & =\left|A_{i} A_{i+1}\right|, \quad i=1,2,3, \\
\left|\underline{u}_{i}+\underline{u}_{i+1}\right| & =\left|B_{i} B_{i+1}\right|, \quad i=1,2,3 .
\end{aligned}
$$

Proof. Iff (2.11) holds, then from

$$
\begin{aligned}
\left|\underline{t}_{1} \underline{t}_{2}+\underline{t}_{2} \underline{t}_{3}+\underline{t}_{3} \underline{t}_{1}\right| & =4 r \rho-\rho^{2}, \\
\left|\underline{u}_{1} \underline{u}_{2}+\underline{u}_{2} \underline{u}_{3}+\underline{u}_{3} \underline{u}_{1}\right| & =4 r_{1} \rho-\rho^{2}
\end{aligned}
$$

it follows that $r=r_{1}$.

Corollary 2.1.3 Let $B_{1} B_{2} B_{3}$ be the degenerate triangle shown in Fig. 1. Then

$$
t_{1}=\sqrt{z^{2}-(r-\rho)^{2}} .
$$


Proof. From (2.5), since $t_{2}=0$, we get

$$
t_{1}^{2}=4 r \rho-\rho^{2} .
$$

Now, using Euler's relation (1.3), we can write

$$
t_{1}^{2}=2 r \rho+2 r \rho-\rho^{2}=z^{2}-r^{2}+2 r \rho-\rho^{2}=z^{2}-(r-\rho)^{2} .
$$

For the following use, the length $\sqrt{z^{2}-(r-\rho)^{2}}$ will be denoted by $t_{0}$, that is

$$
t_{0}=\sqrt{z^{2}-(r-\rho)^{2}} .
$$

See Fig. 2. Let us remark that $t_{0}=\left|P_{1} P_{2}\right|=\left|Q_{1} Q_{2}\right|=\left|P_{1} R_{1}\right|=\left|Q_{1} R_{2}\right|$ since $\left|P_{1} R_{1}\right|=$ $\sqrt{z^{2}-(r-\rho)^{2}}$.

Corollary 2.1.4 For degenerate triangles $P_{1} P_{2} P_{3}$ and $Q_{1} Q_{2} Q_{3}$ shown in Fig. 2 we have

$$
\left|P_{1} P_{2}\right|^{2}=\left|Q_{1} Q_{2}\right|^{2}=\left|P_{1} R_{1}\right|^{2}=\left|Q_{1} R_{2}\right|^{2}=4 r \rho-\rho^{2} .
$$

Proof. Note that $t_{0}^{2}=4 r \rho-\rho^{2}$ holds.

In the following theorem we shall use the length $t_{M}$ given by

$$
t_{M}=\sqrt{(r+z)^{2}-\rho^{2}}
$$

Let us remark that $t_{M} \geq t$ for every tangent drawn from $C_{2}$ to $C_{1}$ (see Fig. 4); $t_{M}=|P Q|$, and $|P Q|=\sqrt{(r+z)^{2}-\rho^{2}}$.

Also, let us remark that $t_{0} \leq t_{1} \leq t_{M}$, where $t_{1}=\left|A_{1} T_{1}\right|$ and $A_{1} A_{2} A_{3}$ is a triangle as noted in Remark 1.

Theorem 2.2 Let $t_{1}$ be such that

$$
t_{0} \leq t_{1} \leq t_{M}
$$

Then the lengths of the other two tangents are given by

$$
t_{2}=\frac{2 r \rho t_{1}+\sqrt{D}}{\rho^{2}+t_{1}^{2}}, \quad t_{3}=\frac{2 r \rho t_{1}-\sqrt{D}}{\rho^{2}+t_{1}^{2}}
$$

where

$$
D=4 r^{2} \rho^{2} t_{1}^{2}-\left(\rho^{2}+t_{1}^{2}\right)\left(\rho^{2} t_{1}^{2}-4 r \rho^{3}+\rho^{4}\right)
$$




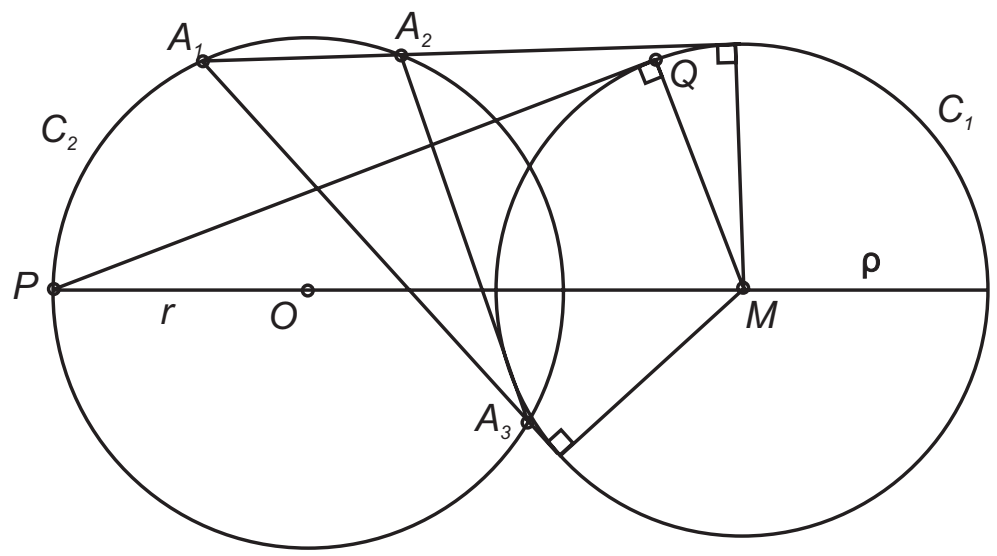

Fig. 4

Proof. The relation (2.9) can be written as

$$
\left(\rho^{2}+t_{1}^{2}\right) t_{2}^{2}-4 r \rho t_{1} t_{2}+\rho^{2} t_{1}^{2}-4 r \rho^{3}+\rho^{4}=0,
$$

from which, solving for $t_{2}$, we get

$$
\left(t_{2}\right)_{1,2}=\frac{2 r \rho t_{1} \pm \sqrt{D}}{\rho^{2}+t_{1}^{2}}
$$

Of course, $\left(t_{2}\right)_{2}=t_{3}$ since

$$
\left|\underline{t}_{1}+\underline{t}_{2}\right|=\left|A_{1} T_{1}\right|, \quad\left|\underline{t}_{1}+\underline{t}_{3}\right|=\left|A_{1} T_{3}\right| .
$$

Thus, it remains to prove that $D \geq 0$ for every $t_{1}$ such that (2.17) holds. For this purpose it is enough to prove that $D=0$ for $t_{1}=t_{M}$ and $t_{1}=-t_{M}$, that is for $t_{1}^{2}=t_{M}^{2}$. The proof is as follows: Putting $t_{M}^{2}$ instead of $t_{1}^{2}$ in $D / \rho^{2}$ and using Euler's relation (1.3) we can write

$$
\begin{aligned}
D / \rho^{2} & =4 r^{2}(r+z)^{2}-4 r^{2} \rho^{2}-(r+z)^{4}+4 r \rho(r+z)^{2} \\
& =4 r^{2}(r+z)^{2}-\left(z^{2}-r^{2}\right)^{2}-(r+z)^{4}+4 r \rho(r+z)^{2} \\
& =(r+z)^{2}\left(4 r^{2}-(z-r)^{2}-(z+r)^{2}+2\left(z^{2}-r^{2}\right)\right)=(r+z)^{2} \cdot 0=0 .
\end{aligned}
$$

Theorem 2.2 is proved.

Although $t_{1}$ is not given explicitly as are $t_{2}$ and $t_{3}$, but by condition $t_{0} \leq t_{1} \leq t_{M}$, it is easy to check that for $t_{1}, t_{2}, t_{3}$ given by (2.17) and (2.18) in the end we get

$$
\left|-t_{1} t_{2}+t_{2} t_{3}-t_{3} t_{1}\right|=\frac{\left(4 r \rho-\rho^{2}\right)\left(\rho^{2}+t_{1}^{2}\right)}{\rho^{2}+t_{1}^{2}}=4 r \rho-\rho^{2} .
$$

Example 1 Let $r=3, z=5, \rho=\frac{8}{3}$. Then

$$
t_{M} \approx 7.542472333, \quad t_{0} \approx 4.988876516 .
$$

If we take $t_{1}=6$, then by (2.18) we get

$$
t_{2} \approx 3.994824489, \quad t_{3} \approx 0.458783759 .
$$

The corresponding triangle $A_{1} A_{2} A_{3}$ is shown in Fig. 4. 


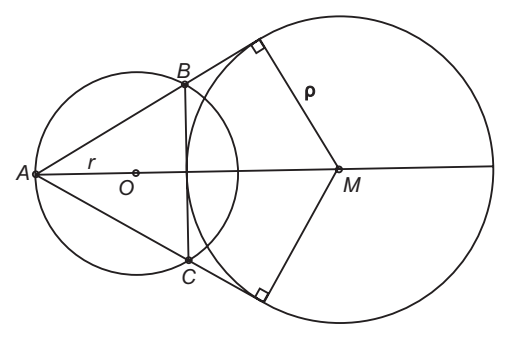

a)

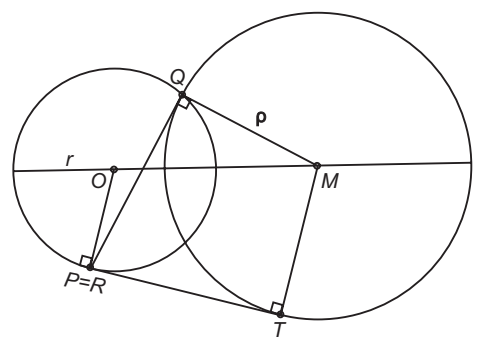

b)

Fig. 5

In connection with this example let us remark that for $t_{1}=t_{M}$ by (2.18), since $D=0$, we have

$$
t_{2}=t_{3}=\frac{2 r \rho t_{M}}{\rho^{2}+t_{M}^{2}} \approx 1.885618083
$$

If we take $t_{1}=t_{0}$, then by (2.18) we have

$$
t_{2}=t_{0}, \quad t_{3}=0 .
$$

In this case, we have $D=4 r^{2} \rho^{2} t_{0}^{2}$ since $\left(\rho^{2}+t_{0}^{2}\right)\left(t_{0}^{2}-4 r \rho+\rho^{2}\right)=0$. Using this example in connection with relation (2.11) we can write

$$
\left|-t_{1} t_{2}+t_{2} t_{3}-t_{3} t_{1}\right| \approx 24.88888889,4 r \rho-\rho^{2} \approx 24.88888889 .
$$

Remark 2 It is easy to see that proving Theorem 2.2 we in fact give another proof of Poncelet's closure theorem for triangles where circles are intersecting, using very simple and elementary facts. Therefore, this theorem may be interesting in itself.

Relation (2.11) which has the key role in the proof of Theorem 2.2 has also an important role in the following theorem.

Theorem 2.3 From (2.11) follows Euler's relation given by (1.3).

Proof. Let $A B C$ be an axially symmetric triangle as shown in Fig. 5a and let $P Q R$ be a degenerate triangle as shown in Fig. $5 \mathrm{~b}$. Then

$$
\begin{aligned}
& t_{1}^{2}=(r+z)^{2}-\rho^{2}, \quad t_{2}^{2}=t_{3}^{2}=r^{2}-(z-\rho)^{2}, \\
& u_{1}^{2}=z^{2}-(r-\rho)^{2}, \quad u_{2}=0, \quad u_{3}=-u_{1} .
\end{aligned}
$$

In connection with $u_{1}$ let us remark that $u_{1}=|P Q|$ and $|P Q|=|P T|$. Theorem 2.3 immediately follows from

$$
\left|\underline{u}_{1} \underline{u}_{2}+\underline{u}_{2} \underline{u}_{3}+\underline{u}_{3} \underline{u}_{1}\right|=4 r \rho-\rho^{2} \quad \text { or } \quad u_{1}^{2}=4 r \rho-\rho^{2}
$$

since

$$
z^{2}-(r-\rho)^{2}=4 r \rho-\rho^{2} \Longleftrightarrow z^{2}-r^{2}=2 r \rho
$$


The following may also be interesting, namely, we can write

$$
-t_{1} t_{2}+t_{2} t_{3}-t_{3} t_{1}=-2 t_{1} t_{2}+t_{2}^{2}, \quad-u_{1} u_{2}+u_{2} u_{3}-u_{3} u_{1}=-u_{1}^{2},
$$

and by (2.11) it holds

$$
-2 t_{1} t_{2}+t_{2}^{2}=-u_{1}^{2}
$$

or

$$
4 t_{1}^{2} t_{2}^{2}=\left(t_{2}^{2}+u_{1}^{2}\right)^{2}
$$

which can be written as

$$
\left(r^{2}+2 r \rho-z^{2}\right)(r+z-\rho)^{2}=0 .
$$

Let us remark that from $z^{2}-r^{2}=2 r \rho$, putting $r+z=\rho$, we get $z=3 r$ and that for $z=3 r, \rho=4 r$ it holds $z^{2}-r^{2}=2 r \rho$. In this limit case we have $4 r \rho-\rho^{2}=0$. Thus in this case, $t_{1}=t_{2}=t_{3}=0$ (the triangle becomes tangential point of $C_{1}$ and $C_{2}$ ).

\section{Some relations concerning bicentric quadrilaterals when excircles instead of incircles are under consideration}

Notation used:

Let $r, \rho$ and $z$ be any given lengths (positive numbers) such that

$$
z^{2}=r^{2}+\rho^{2}+\sqrt{4 r^{2} \rho^{2}+\rho^{4}}
$$

Let $M$ and $O$ be points and $C_{1}$ and $C_{2}$ be circles such that

$$
|M O|=z, \quad C_{1}=M(\rho), \quad C_{2}=O(r) .
$$

The circles $C_{1}$ and $C_{2}$ are not intersecting since from (3.1) it follows that

$$
z^{2}>r^{2}+\rho^{2}+2 r \rho \text { or } z>r+\rho
$$

Let us remark that (3.1) follows from Fuss' relation (1.1), namely, from

$$
\left(r^{2}-z^{2}\right)^{2}=2 \rho^{2}\left(r^{2}+z^{2}\right)
$$

it follows that

$$
z^{2}=r^{2}+\rho^{2} \pm \sqrt{4 r^{2} \rho^{2}+\rho^{4}}
$$

The condition for a bicentric quadrilateral where $C_{1}$ is inside of $C_{2}$ is given by

$$
z^{2}=r^{2}+\rho^{2}-\sqrt{4 r^{2} \rho^{2}+\rho^{4}}
$$

from which it follows that $z<r-\rho$. 


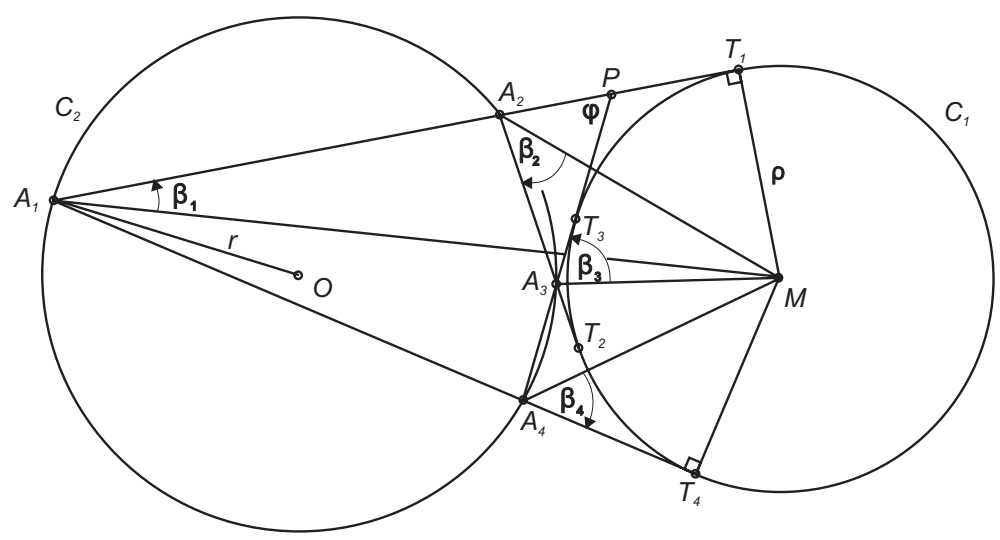

Fig. 6

Now, for example, let $r=4, \rho=3, z=7.115617418$ (see Fig. 6). It is easy to see that

$$
\left(t_{1}-t_{2}+t_{3}-t_{4}\right) \rho=\text { area of quadrilateral } A_{1} A_{2} A_{3} A_{4},
$$

where

$$
\left|A_{1} A_{2}\right|=t_{1}-t_{2}, \quad\left|A_{2} A_{3}\right|=t_{2}-t_{3}, \quad\left|A_{3} A_{4}\right|=t_{4}-t_{3}, \quad\left|A_{4} A_{1}\right|=t_{1}-t_{4} .
$$

Thus, in this case, we must instead of $t_{2}$ and $t_{4}$ take $-t_{2}$ and $-t_{4}$. It is because we must use oriented angles. Namely, if the angle $M A_{i} T_{i}, i=1,2,3,4$, is negatively oriented, then instead of $t_{i}$ we must take $-t_{i}$.

It is easy to see that for every quadrilateral $A_{1} A_{2} A_{3} A_{4}$ whose excircle is $C_{1}$ and circumcircle is $C_{2}$ either

$$
t_{1},-t_{2}, t_{3},-t_{4}
$$

or

$$
-t_{1}, t_{2},-t_{3}, t_{4}
$$

holds. Namely, the angles $M A_{1} T_{1}$ and $M A_{3} T_{3}$ are positively oriented and the angles $M A_{2} T_{2}$ and $M A_{4} T_{4}$ are negatively oriented or it is conversely.

Also, it can be easily seen that

$$
\left|\underline{t}_{i}+\underline{t}_{i+1}\right|=\left|A_{i} A_{i+1}\right|, \quad i=1,2,3,4,
$$

where

$$
\begin{aligned}
& \underline{t}_{i}=t_{i} \text { if } \angle M A_{i} T_{i} \text { is positively oriented, } \\
& \underline{t}_{i}=-t_{i} \text { if } \angle M A_{i} T_{i} \text { is negatively oriented. }
\end{aligned}
$$

Using vertices $A_{1}, A_{2}, A_{3}, A_{4}$ instead of $T_{1}, T_{2}, T_{3}, T_{4}$ this can be expressed as follows:

$$
\begin{aligned}
& \underline{t}_{i}=t_{i} \text { if } \angle M A_{i} A_{i+1} \text { is positively oriented, } \\
& \underline{t}_{i}=-t_{i} \text { if } \angle M A_{i} A_{i+1} \text { is negatively oriented. }
\end{aligned}
$$


Of course, if $\angle M A_{i} A_{i+1}$ is "obtuse" then its supplement is taken. Now, using Fig. 6, we shall prove that

$$
\beta_{1}-\beta_{2}+\beta_{3}-\beta_{4}=0^{\circ}
$$

where

$$
\beta_{i}=\text { measure of } \angle M A_{i} T_{i}, \quad i=1,2,3,4 .
$$

First from triangle $P A_{1} A_{4}$, since the measure of $\angle A_{3} A_{4} T_{4}=2 \beta_{4}$, we have

$$
2 \beta_{4}=2 \beta_{1}+\varphi
$$

Now, from triangle $P A_{2} A_{3}$ we see that

$$
\varphi+2 \beta_{2}+\left(180-2 \beta_{3}\right)=180^{\circ}
$$

From (3.8) and (3.9) follows (3.7).

Before we state the following theorem we shall prove that

$$
\left(t_{1}-t_{2}+t_{3}-t_{4}\right) \rho^{2}=-t_{1} t_{2} t_{3}+t_{2} t_{3} t_{4}-t_{3} t_{4} t_{1}+t_{4} t_{1} t_{2} .
$$

Starting from (3.7) we can write

$$
\tan \left(\beta_{1}+\beta_{3}\right)=\tan \left(\beta_{2}+\beta_{4}\right)
$$

from which, using the relation

$$
\frac{\rho}{t_{i}}=\tan \beta_{i}, \quad i=1,2,3,4
$$

we readily get (3.10).

Theorem 3.1 Let $A_{1} A_{2} A_{3} A_{4}$ be a bicentric quadrilateral whose excircle is $C_{1}$ and circumcircle is $C_{2}$, where $C_{1}$ and $C_{2}$ are given by (3.2). Then

$$
t_{1} t_{3}=t_{2} t_{4}=\rho^{2}
$$

where

$$
t_{i}=\left|A_{i} T_{i}\right|, \quad i=1,2,3,4
$$


Proof. Since either (3.5) or (3.6) is possible we may assume without loss of generality that (3.5) is valid, namely, that the situation is like that in Fig. 6, where

$$
\left|A_{1} A_{2}\right|=t_{1}-t_{2}, \quad\left|A_{2} A_{3}\right|=t_{2}-t_{3}, \quad\left|A_{3} A_{4}\right|=t_{4}-t_{3}, \quad\left|A_{4} A_{1}\right|=t_{1}-t_{4} .
$$

Since (3.4) holds we have the equality

$$
\left(t_{1}-t_{2}+t_{3}-t_{4}\right) \rho=\sqrt{\left(t_{1}-t_{2}\right)\left(t_{2}-t_{3}\right)\left(t_{4}-t_{3}\right)\left(t_{1}-t_{4}\right)}
$$

or

$$
\left(t_{1}-t_{2}+t_{3}-t_{4}\right)^{2} \rho^{2}=\left(t_{1}-t_{2}\right)\left(t_{2}-t_{3}\right)\left(t_{4}-t_{3}\right)\left(t_{1}-t_{4}\right) .
$$

The above equality, using equality (3.10), can be written as

$$
\left(t_{1}-t_{2}+t_{3}-t_{4}\right)\left(-t_{1} t_{2} t_{3}+t_{2} t_{3} t_{4}-t_{3} t_{4} t_{1}+t_{4} t_{1} t_{2}\right)=\left(t_{1}-t_{2}\right)\left(t_{2}-t_{3}\right)\left(t_{4}-t_{3}\right)\left(t_{1}-t_{4}\right)
$$

or

$$
t_{1}^{2} t_{3}^{2}-2 t_{1} t_{2} t_{3} t_{4}+t_{2}^{2} t_{4}^{2}=0
$$

from which it follows that $\left(t_{1} t_{3}-t_{2} t_{4}\right)^{2}=0$ or

$$
t_{1} t_{3}=t_{2} t_{4}
$$

Now, from (3.10), putting $t_{4}=\frac{t_{1} t_{3}}{t_{2}}$, we get

$$
\rho^{2}=\frac{t_{1} t_{3}\left(t_{1}+t_{2}\right)\left(t_{2}+t_{3}\right)}{\left(t_{1}+t_{2}\right)\left(t_{2}+t_{3}\right)}=t_{1} t_{3}
$$

Also it is valid $\rho^{2}=t_{2} t_{4}$ since (3.14) is valid. Theorem 3.1 is proved.

Corollary 3.1.1 Let $A_{1} A_{2} A_{3} A_{4}$ be any given tangential quadrilateral whose excircle is $C_{1}$. Then this quadrilateral will be a bicentric one whose circumcircle is $C_{2}$ iff (3.12) holds.

Proof. From (3.10) and (3.12) follows (3.13).

Theorem 3.2 Let ABCD and PRQS be two bicentric quadrilaterals such that their excircles are congruent. Then their circumcircles are also congruent iff

$$
t_{1} t_{2}+t_{2} t_{3}+t_{3} t_{4}+t_{4} t_{1}=u_{1} u_{2}+u_{2} u_{3}+u_{3} u_{4}+u_{4} u_{1}
$$

where $t_{i}$ and $u_{i}, i=1,2,3,4$, are the lengths of the consecutive tangents relating to $A B C D$ and $P Q R S$, respectively. 
Proof. First, let us remark, that from (3.5) and also from (3.6) it follows that

$$
\underline{t}_{1} \underline{t}_{2}+\underline{t}_{2} \underline{t}_{3}+\underline{t}_{3} \underline{t}_{4}+\underline{t}_{4} \underline{t}_{1}=-t_{1} t_{2}-t_{2} t_{3}-t_{3} t_{4}-t_{4} t_{1}
$$

where $\underline{t}_{i}=t_{i}$ or $\underline{t}_{i}=-t_{i}$ depending on how the angle $M A_{i} T_{i}$ is oriented. Using the expression $-\left(t_{1} t_{2}+t_{2} t_{3}+t_{3} t_{4}+t_{4} t_{1}\right)$ and the equalities $t_{1} t_{3}=\rho^{2}$ and $t_{2} t_{4}=\rho^{2}$ given by (3.12), we find that

$$
-\left(t_{1} t_{2}+t_{2} t_{3}+t_{3} t_{4}+t_{4} t_{1}\right)=\frac{t_{1}^{2} t_{2}^{2}+\rho^{2}\left(t_{1}^{2}+t_{2}^{2}\right)+\rho^{4}}{-t_{1} t_{2}} .
$$

Let $r$ be the radius of the circumcircle of $A B C D$. We have to prove that $r$ is also the radius of the circumcircle of PQRS iff (3.15) holds. In the proof we shall use the well-known relations concerning chordal quadrilaterals. These relations are

$$
r^{2}=\frac{(a d+c d)(a c+b d)(a d+b c)}{16 J^{2}}, \quad J^{2}=a b c d
$$

where

$$
a=t_{1}-t_{2}, \quad b=t_{2}-t_{3}, \quad c=t_{4}-t_{3}, \quad d=t_{1}-t_{4}, \quad J=\text { area of } A B C D .
$$

From (3.17) it follows that

$$
16 r^{2}=a^{2}+b^{2}+c^{2}+d^{2}+\frac{a b c}{d}+\frac{b c d}{a}+\frac{c d a}{b}+\frac{d a b}{c}
$$

which, using (3.12), can be written as

$$
16 r^{2} \rho^{2}+4 \rho^{4}=\left[\frac{t_{1}^{2} t_{2}^{2}+\rho^{2}\left(t_{1}^{2}+t_{2}^{2}\right)+\rho^{4}}{-t_{1} t_{2}}+2 \rho^{2}\right]^{2} .
$$

Analogously, for the bicentric quadrilateral $P Q R S$ we have

$$
16 r_{1}^{2} \rho^{2}+4 \rho^{4}=\left[\frac{u_{1}^{2} u_{2}^{2}+\rho^{2}\left(u_{1}^{2}+u_{2}^{2}\right)+\rho^{4}}{-u_{1} u_{2}}+2 \rho^{2}\right]^{2},
$$

where $r_{1}$ is the radius of the circumcircle of PQRS. Thus, iff (3.15) is valid, then $r_{1}=r$. Theorem 3.2 is proved.

Now, we shall prove that the left-hand side of (3.18) can be written as $4\left(r^{2}+\rho^{2}-z^{2}\right)^{2}$, namely, that it holds

$$
16 r^{2} \rho^{2}+4 \rho^{4}=4\left(r^{2}+\rho^{2}-z^{2}\right)^{2} .
$$

For this purpose, we shall add $\rho^{4}+2 r^{2} \rho^{2}-2 \rho^{2} z^{2}$ on both sides of Fuss' relation for a bicentric quadrilateral

$$
2 \rho^{2}\left(r^{2}+z^{2}\right)=\left(r^{2}-z^{2}\right)^{2}
$$




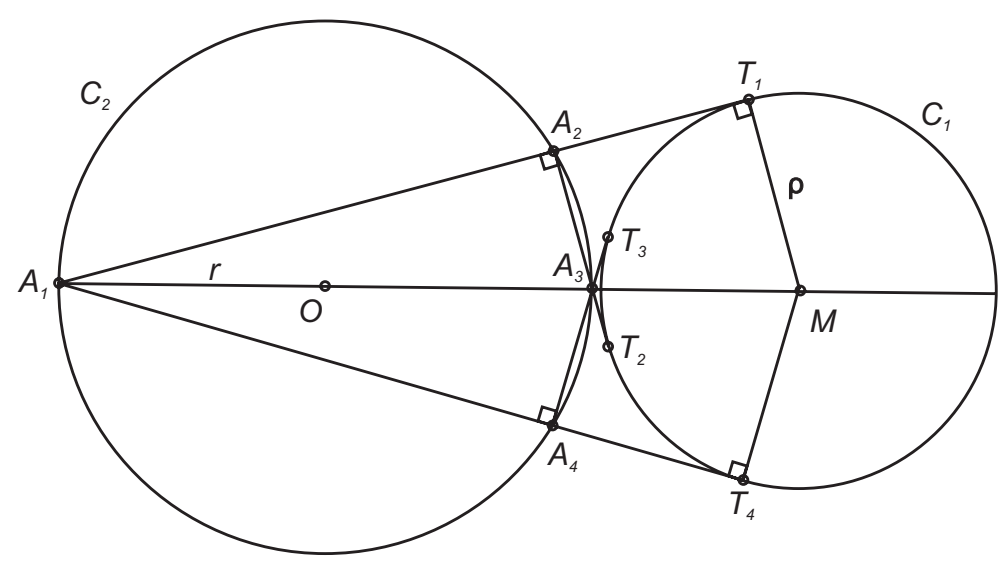

Fig. 7

So, we can write

$$
2 \rho^{2}\left(r^{2}+z^{2}\right)+\left(\rho^{4}+2 r^{2} \rho^{2}-2 \rho^{2} z^{2}\right)=\left(r^{2}-z^{2}\right)^{2}+\left(\rho^{4}+2 r^{2} \rho^{2}-2 \rho^{2} z^{2}\right)
$$

or

$$
4 r^{2} \rho^{2}+\rho^{4}=\left(r^{2}+\rho^{2}-z^{2}\right)^{2} .
$$

Thus, the equality (3.18) can be written as

$$
\frac{t_{1}^{2} t_{2}^{2}+\rho^{2}\left(t_{1}^{2}+t_{2}^{2}\right)+\rho^{4}}{-t_{1} t_{2}}=2\left(r^{2}-z^{2}\right)
$$

or

$$
\frac{t_{1}^{2} t_{2}^{2}+\rho^{2}\left(t_{1}^{2}+t_{2}^{2}\right)+\rho^{4}}{t_{1} t_{2}}=2\left(z^{2}-r^{2}\right)
$$

Since (3.16) holds, we have the following relation

$$
t_{1} t_{2}+t_{2} t_{3}+t_{3} t_{4}+t_{4} t_{1}=2\left(z^{2}-r^{2}\right)
$$

In some of the following theorems we shall use the relations

$$
t_{m}=\sqrt{(z-r)^{2}-\rho^{2}}, \quad t_{M}=\sqrt{(z+r)^{2}-\rho^{2}} .
$$

See Fig. 7. As can be seen, $t_{m}=\left|A_{3} T_{3}\right|$ is the length of the shortest tangent that can be drawn from $C_{2}$ to $C_{1}$, and $t_{M}=\left|A_{1} T_{1}\right|$ is the length of the largest tangent that can be drawn from $C_{2}$ to $C_{1}$.

By (3.12) it holds

$$
t_{m} t_{M}=\rho^{2} .
$$

Theorem 3.3 From (3.22) follows Fuss' relation given by (1.1). 
Proof. It holds

$$
t_{m}^{2} t_{M}^{2}=\left(r^{2}-z^{2}\right)^{2}-2 \rho^{2}\left(r^{2}+z^{2}\right)+\rho^{4},
$$

and from (3.22) it follows $t_{m}^{2} t_{M}^{2}-\rho^{4}=0$, that is

$$
\left(r^{2}-z^{2}\right)^{2}-2 \rho^{2}\left(r^{2}+z^{2}\right)=0 .
$$

Theorem 3.3 is proved.

Thus, in this way we can deduce Fuss' relation for bicentric quadrilaterals.

Fuss' relation for bicentric quadrilaterals is closely connected with the relations (3.12) and (3.20). So, for example, using Fig. 7, it is easy to show that (3.20) holds for

$$
t_{1}=t_{M}, \quad t_{2}=\rho, \quad t_{3}=t_{m}, \quad t_{4}=\rho .
$$

First, let us remark that from $t_{2} t_{4}=\rho^{2}$, since $t_{2}=t_{4}$ and (3.12) holds, it follows that $t_{2}=\rho$. So, in this case, we have

$$
t_{1} t_{2}+t_{2} t_{3}+t_{3} t_{4}+t_{4} t_{1}=2 \rho\left(t_{m}+t_{M}\right)
$$

and it is easy to show that

$$
2 \rho\left(t_{m}+t_{M}\right)=2\left(z^{2}-r^{2}\right) .
$$

Namely, since $2 t_{m} t_{M}=2 \rho^{2}$, we can write

$$
\rho^{2}\left(t_{m}+t_{M}\right)^{2}=\rho^{2}\left[(z-r)^{2}+(z+r)^{2}-2 \rho^{2}\right]+2 \rho^{4}=2 \rho^{2}\left(r^{2}+z^{2}\right) .
$$

Thus,

$$
\left[2 \rho\left(t_{m}+t_{M}\right)\right]^{2}=\left[2\left(z^{2}-r^{2}\right)\right]^{2},
$$

since $2 \rho^{2}\left(r^{2}+z^{2}\right)=\left(z^{2}-r^{2}\right)^{2}$ by Fuss' relation (1.1).

Also, using Fuss' relation, it can be easily shown that the following theorem holds.

Theorem 3.4 It holds

$$
\begin{gathered}
(z+r)^{2} t_{m}=(z-r)^{2} t_{M}, \\
t_{m}=\frac{z-r}{z+r} \rho, \quad t_{M}=\frac{z+r}{z-r} \rho, \\
t_{m}=\frac{z^{2}-r^{2}-\sqrt{D}}{2 \rho}, \quad t_{M}=\frac{z^{2}-r^{2}+\sqrt{D}}{2 \rho},
\end{gathered}
$$

where

$$
D=\left(z^{2}-r^{2}\right)^{2}-4 \rho^{4}
$$


Proof. The proof that (3.24) holds:

$$
(z+r)^{4} t_{m}^{2}-(z-r)^{4} t_{M}^{2}=4 r z\left[\left(z^{2}-r^{2}\right)^{2}-2 \rho^{2}\left(z^{2}+r^{2}\right)\right]=4 r z \cdot 0=0 .
$$

Concerning (3.25), it is easy to show that

$$
\begin{aligned}
& \left(r^{2}-z^{2}\right)^{2}=2 \rho^{2}\left(r^{2}+z^{2}\right) \Longleftrightarrow \sqrt{(r-z)^{2}-\rho^{2}}=\frac{r-z}{r+z} \rho, \\
& \left(r^{2}-z^{2}\right)^{2}=2 \rho^{2}\left(r^{2}+z^{2}\right) \Longleftrightarrow \sqrt{(r+z)^{2}-\rho^{2}}=\frac{r+z}{r-z} \rho .
\end{aligned}
$$

So, from

$$
(r-z)^{2}-\rho^{2}=\left(\frac{r-z}{r+z}\right)^{2} \rho^{2}
$$

it follows

$$
\left(r^{2}-z^{2}\right)^{2}=\rho^{2}\left((r-z)^{2}+(r+z)^{2}\right)
$$

or

$$
\left(r^{2}-z^{2}\right)^{2}=2 \rho^{2}\left(r^{2}+z^{2}\right)
$$

Obviously, the converse is also valid. Concerning (3.26), using (3.22) and (3.23), we can write

$$
t_{m} t_{M}=\rho^{2}, \quad t_{m}+t_{M}=\frac{z^{2}-r^{2}}{\rho}
$$

from which (3.26) follows.

Corollary 3.4.1 The following is true:

$$
z^{2}>r^{2}+2 \rho^{2}
$$

Proof. It follows from (3.27). Of course, it also follows from (3.1) since $\sqrt{4 r^{2} \rho^{2}+\rho^{4}}>$ $\rho^{2}$.

\section{Theorem 3.5 It holds}

$$
A\left(t_{1},-t_{2}, t_{3},-t_{4}\right) \cdot H\left(t_{1},-t_{2}, t_{3},-t_{4}\right)=\rho^{2},
$$

where $A\left(t_{1},-t_{2}, t_{3},-t_{4}\right)$ and $H\left(t_{1},-t_{2}, t_{3},-t_{4}\right)$ are the arithmetic and harmonic means of $t_{1},-t_{2}, t_{3},-t_{4}$.

Proof. (3.12), $t_{1} t_{3}=t_{2} t_{4}=\rho^{2}$, implies $t_{1} t_{2} t_{3} t_{4}=\rho^{4}$. If we divide equation (3.10) by $t_{1} t_{2} t_{3} t_{4}$, we can write

$$
\frac{\left(t_{1}-t_{2}+t_{3}-t_{4}\right) \rho^{2}}{\rho^{4}}=\frac{-t_{1} t_{2} t_{3}+t_{2} t_{3} t_{4}-t_{3} t_{4} t_{1}+t_{4} t_{1} t_{2}}{t_{1} t_{2} t_{3} t_{4}}
$$

or

$$
\frac{t_{1}-t_{2}+t_{3}-t_{4}}{4} \cdot \frac{4}{\frac{1}{t_{1}}-\frac{1}{t_{2}}+\frac{1}{t_{3}}-\frac{1}{t_{4}}}=\rho^{2} .
$$

Theorem 3.5 is proved. 
Theorem 3.6 Let $A B C D$ be any given bicentric quadrilateral whose excircle is $C_{1}$ and circumcircle is $C_{2}$, where $C_{1}$ and $C_{2}$ are given by (3.2). Then

$$
e f=2\left(z^{2}-r^{2}-2 \rho^{2}\right)
$$

where $e=|A C|, f=|B D|$. In other words, for every bicentric quadrilateral whose excircle is $C_{1}$ and circumcircle is $C_{2}$, the product of the lengths of its diagonals is the constant $2\left(z^{2}-r^{2}-2 \rho^{2}\right)$.

Proof. Let $a=t_{1}-t_{2}, b=t_{2}-t_{3}, c=t_{4}-t_{3}, d=t_{1}-t_{4}$ be the lengths of the sides of $A B C D$. Then, by Ptolomy's theorem,

$$
e f=a c+b d
$$

and we can write

$$
\begin{aligned}
a c+b d & =\left(t_{1}-t_{2}\right)\left(t_{4}-t_{3}\right)+\left(t_{2}-t_{3}\right)\left(t_{1}-t_{4}\right) \\
& =\left(t_{1} t_{2}+t_{2} t_{3}+t_{3} t_{4}+t_{4} t_{1}\right)-2\left(t_{1} t_{3}+t_{2} t_{4}\right) \\
& =2\left(z^{2}-r^{2}\right)-2\left(\rho^{2}+\rho^{2}\right)=2\left(z^{2}-r^{2}-2 \rho^{2}\right) .
\end{aligned}
$$

It is easy to see that we have the same result if instead of the possibility (3.5) we take the possibility (3.6). Theorem 3.6 is proved.

Theorem 3.7 Let $r, \rho$ and $z$ be any given positive numbers such that (1.1) is satisfied, and let $t_{m}$ and $t_{M}$ be given by (3.21). Then every positive solution $\left(t_{1}, t_{2}, t_{3}, t_{4}\right) \in \mathbb{R}_{+}^{4}$ of the equations

$$
t_{1} t_{2}+t_{2} t_{3}+t_{3} t_{4}+t_{4} t_{1}=2\left(z^{2}-r^{2}\right), \quad t_{1} t_{3}=\rho^{2}, \quad t_{2} t_{4}=\rho^{2}
$$

is given by

$t_{1}$ is a positive number such that $t_{m} \leq t_{1} \leq t_{M}$,

$$
\begin{gathered}
t_{2}=\frac{\left(z^{2}-r^{2}\right) t_{1}+\sqrt{D}}{\rho^{2}+t_{1}^{2}}, \\
t_{3}=\frac{\rho^{2}}{t_{1}}, \\
t_{4}=\frac{\rho^{2}}{t_{2}},
\end{gathered}
$$

where

$$
D=\left(z^{2}-r^{2}\right)^{2} t_{1}^{2}-\rho^{2}\left(\rho^{2}+t_{1}^{2}\right)^{2}
$$


Proof. The equation $t_{1} t_{2}+t_{2} t_{3}+t_{3} t_{4}+t_{4} t_{1}=2\left(z^{2}-r^{2}\right)$, using equations $t_{1} t_{3}=\rho^{2}$ and $t_{2} t_{4}=\rho^{2}$, can be written as

$$
\left(\rho^{2}+t_{1}^{2}\right) t_{2}^{2}-2\left(z^{2}-r^{2}\right) t_{1} t_{2}+\rho^{2}\left(t_{1}^{2}+\rho^{2}\right)=0
$$

from which it follows that

$$
\left(t_{2}\right)_{1,2}=\frac{\left(z^{2}-r^{2}\right) t_{1} \pm \sqrt{D}}{\rho^{2}+t_{1}^{2}}
$$

It is unessential which of $\left(t_{2}\right)_{1}$ and $\left(t_{2}\right)_{2}$ will be taken for $t_{2}$ since

$$
\frac{\rho^{2}}{\left(t_{2}\right)_{1}}=\frac{\rho^{2}\left(\rho^{2}+t_{1}^{2}\right)}{\left(z^{2}-r^{2}\right) t_{1}+\sqrt{D}}=\frac{\left(z^{2}-r^{2}\right) t_{1}-\sqrt{D}}{\rho^{2}+t_{1}^{2}}=\left(t_{2}\right)_{2} .
$$

If we take $t_{2}=\left(t_{2}\right)_{1}$, then $\frac{\rho^{2}}{t_{2}}=\left(t_{2}\right)_{2}$, that is, by (3.33), $\left(t_{2}\right)_{2}=t_{4}$. But if we take $t_{2}=\left(t_{2}\right)_{2}$, then $\frac{\rho^{2}}{t_{2}}=\left(t_{2}\right)_{1}$. Thus, in this case $\left(t_{2}\right)_{1}=t_{4}$.

Now, since in the expression of $t_{2}$ in (3.31) appears the term $\sqrt{D}$, we have to prove that $D \geq 0$ for every $t_{1}$ such that $t_{m} \leq t_{1} \leq t_{M}$. Of course, for this purpose it suffices to prove that $D=0$ for $t_{1}=t_{m}$ and $t_{1}=t_{M}$.

It is easy to show that

$$
\begin{aligned}
\left(z^{2}-r^{2}\right)^{2} t_{m}^{2}-\rho^{2}\left(\rho^{2}+t_{m}^{2}\right)^{2}=0 \Longleftrightarrow(1.1), \\
\left(z^{2}-r^{2}\right)^{2} t_{M}^{2}-\rho^{2}\left(\rho^{2}+t_{M}^{2}\right)^{2}=0 \Longleftrightarrow(1.1),
\end{aligned}
$$

where (1.1) stands instead of Fuss' relation given by (1.1). So, for $t_{1}=t_{m}$, we can write

$$
\left(z^{2}-r^{2}\right)^{2} t_{m}^{2}-\rho^{2}\left(\rho^{2}+t_{m}^{2}\right)^{2}=(z-r)^{2}\left[\left(z^{2}-r^{2}\right)^{2}-2 \rho^{2}\left(z^{2}+r^{2}\right)\right]=(z-r)^{2} \cdot 0=0 .
$$

This completes the proof of Theorem 3.7.

Although $t_{1}$ is not given explicitly but by condition $t_{m} \leq t_{1} \leq t_{M}$, it is easy to check that for $t_{1}, t_{2}, t_{3}, t_{4}$ given by (3.30)-(3.33) in the end we get

$$
t_{1} t_{2}+t_{2} t_{3}+t_{3} t_{4}+t_{4} t_{1}=\frac{\left(z^{2}-r^{2}\right) t_{1}+\sqrt{D}}{t_{1}}+\frac{\left(z^{2}-r^{2}\right) t_{1}-\sqrt{D}}{t_{1}}=2\left(z^{2}-r^{2}\right) .
$$

Corollary 3.7.1 Let $C_{1}$ and $C_{2}$ be circles such that (3.1) and (3.2) holds. Let $A_{1}$ be any given point on $C_{2}$ and let $t_{1}$ be the length of the tangent $A_{1} T_{1}$ drawn from $C_{2}$ to $C_{1}$. Then the lengths $t_{2}, t_{3}, t_{4}$ of the other three tangents drawn from $C_{2}$ to $C_{1}$ are given by (3.31), (3.32) and (3.33).

Here is an example: 


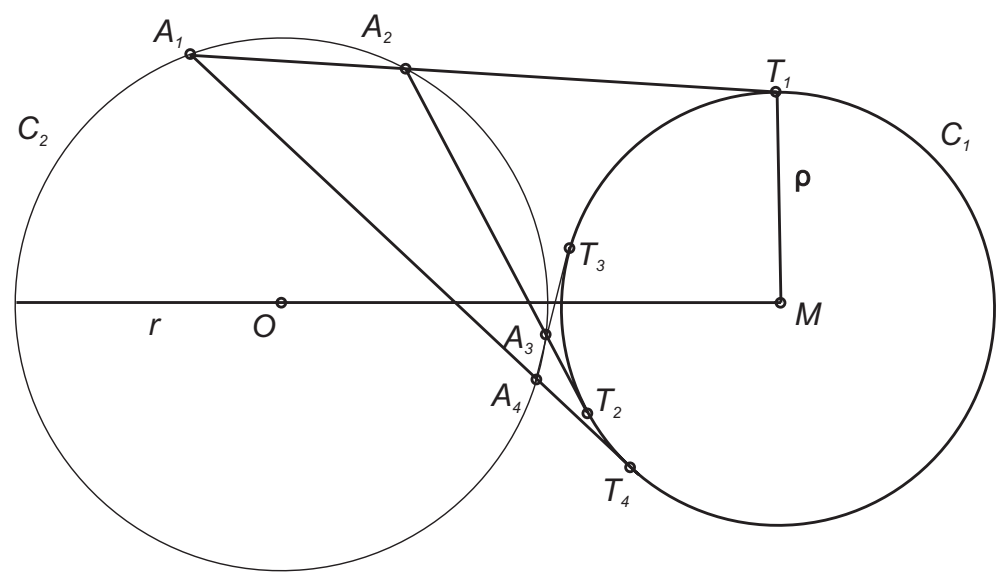

Fig. 8

Example 2 Let $r=4, \rho=3, z=7.115617418$. Then

$$
t_{m} \approx 0.840875671, \quad t_{M} \approx 10.70312807, \quad D \approx 28799.07696 .
$$

If we take $t_{1}=8$, then

$$
t_{2} \approx 6.119986271, \quad t_{3}=1.125, \quad t_{4} \approx 1.470591534 .
$$

The corresponding quadrilateral $A_{1} A_{2} A_{3} A_{4}$ is shown in Fig. 8.

It can be checked that

$$
t_{1} t_{2}+t_{2} t_{3}+t_{3} t_{4}+t_{4} t_{1} \approx 69.26402247=2\left(z^{2}-r^{2}\right) .
$$

Also, it can be checked that

$$
\begin{gathered}
\beta_{1} \approx 20.55604522^{\circ}, \quad \beta_{2} \approx 26.11396343^{\circ}, \\
\beta_{3} \approx 69.44395478^{\circ}, \quad \beta_{4} \approx 63.88603657^{\circ}, \\
\beta_{1}-\beta_{2}+\beta_{3}-\beta_{4}=0^{\circ},
\end{gathered}
$$

where $\beta_{i}=\arctan \frac{\rho}{t_{i}}, i=1,2,3,4$.

If in this figure we write $A_{2}$ where is $A_{4}$ and $A_{4}$ where is $A_{2}$, then the angles $M A_{1} T_{1}$ and $M A_{3} T_{3}$ will be negatively oriented and in this case will be

$$
-\beta_{1}+\beta_{2}-\beta_{3}+\beta_{4}=0^{\circ} .
$$

Remark 3 As can be seen, by proving Theorem 3.7, we in fact give another proof of Poncelet's closure theorem for bicentric quadrilaterals, when the excircle instead of the incircle is under consideration. In this proof, we use very simple and elementary mathematical facts. Therefore, this proof may be interesting in itself. 


\section{References}

[1] Barth, W.; Bauer, Th.: Poncelet's Theorem. Exposition. Math. 14 (1996), 125-144.

[2] Bos, H.J.M.; Kers, C.; Oort, F.; Raven, D.W.: Poncelet's closure theorem. Exposition. Math. 5 (1987), 289-364.

[3] Bos, H.J.M.: The closure theorem of Poncelet. Rend. Sem. Mat. Fis. Milano 54 (1984), 145-158.

[4] Dörrie, H.: 100 Great Problems of Elementary Mathematics, Their History and Solution. Dover Publications, Inc., 1965 (Originally published in German under the title of Triumph der Mathematik).

[5] Fuss, N.: De quadrilateris quibus circulum tam inscribere quam circumscribere licet. Nova acta acad. sci. Petrop. 10, St. Petersburg (1797), 103-125.

[6] Griffiths, P.; Harris, J.: On Cayley's explicit solution to Poncelet's porism. Enseign. Math. (1978), 31-40.

[7] Hraskó, A.: Poncelet-type Problems, an Elementary Approach. Elem. Math. 55 (2000), 45-62.

[8] Herr, U.: Über das Theorem von Poncelet. Staatsexamensarbeit vorgelegt dem Fachbereich Mathematik, Johannes Gutenberg-Universität Mainz 2000.

[9] Poncelet, J.W.: Traité des propriétés projectives des figures. Paris 1865 (first ed. in 1822).

[10] Radić, M.: Some relations and properties concerning tangential polygons. Math. Commun. 4 (1999), 197-206.

Mirko Radić

University of Rijeka

Faculty of Philosophy

Department of Mathematics

Omladinska 14

51000 Rijeka, Croatia

e-mail: mradic@mapef .pefri.hr 